\title{
0 uso do aplicativo ANKI no estudo da termodinâmica no Ensino Superior: um relato de experiência
}

DOI: https://doi.org/10.35168/2176-896X.UTP.Tuiuti.2021.Vol7.N63.pp131-149

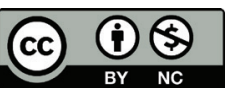

Igor Lindemberg Ventura Gonçalves igorvenlg@gmail.com 


\section{0 uso do aplicativo ANKI no estudo da termodinâmica no ensino superior: um relato de experiência}

\section{Resumo}

Atualmente os smartphones estão cada vez mais cheios de tecnologias e ferramentas de inúmeras utilidades. Existem aplicativos com diversas finalidades, inclusive educativas. Essas propriedades dos smartphones os tornam recursos valiosos na implementação do ensino de Física interativo e contemporâneo às tecnologias atuais. Neste artigo relatamos a nossa experiência e as nossa percepções sobre a inserção do aplicativo ANKI no ensino de Termodinâmica, em uma turma do curso de Agronomia, da Universidade Federal do Cariri (UFCA), localizada no interior do Ceará. O nosso objetivo com a inserção do aplicativo era de avaliar seu potencial quanto ao estímulo e a mudança na rotina de estudo dos alunos. Além disso, tínhamos como propósito apresentar aos alunos uma forma uniforme e contínua de estudar, sem picos de stress pré-teste, e de avaliar a percepção dos alunos sobre a inserção do aplicativo na rotina de estudo. Após a análise do questionário aplicado verificamos que o aplicativo ANKI é um recurso potencialmente eficaz quanto à motivação dos alunos, a assimilação dos conteúdos de Termodinâmica e ao estabelecimento de uma rotina contínua e uniforme de estudo.

Palavras-chave: Smartphones. ANKI. Termodinâmica. 


\section{The use of ANKI application in the study of thermodynamics in Higher Education: an experience report}

\section{Abstract}

Nowadays smartphones are increasingly full of technologies and tools of numerous uses. There are apps for various purposes, including educational. These properties of smartphones make them valuable resources in the implementation of interactive and contemporary Physics teaching to current tecnology. In this article we report our experience and our perceptions about the insertion of the ANKI application in the teaching of Thermodynamics, in a class of the Agronomy course, at the Federal University of Cariri (UFCA), located in the interior of Ceará. Our objective with the insertion of the app was to assess its potential in terms of stimulating and changing the students' study routine. In addition, our purpose was to present students with a uniform and continuous way of studying, without peaks of pre-test stress, and to assess students' perception of the application's insertion in their study routine. After analyzing the applied questionnaire, we verified that the ANKI application is a potentially effective resource regarding student motivation, the assimilation of thermodynamics contents and the establishment of a continuous and uniform study routine.

Keywords: Smartphones. ANKI. Thermodynamics 


\section{0 uso do aplicativo ANKI no estudo da termodinâmica no ensino superior: um relato de experiência}

\section{Introdução}

As tecnologias da Informação e Comunicação (TIC) compreendem os recursos tecnológicos que viabilizam a transmissão da informação através de diferentes meios de comunicação. Em particular, no ensino de Física, o surgimento das TICs tem provocado diversas implicações no estudo teórico do processo de ensino e aprendizagem nos últimos tempos (NUNES; SILVA; MERCADO, 2016; SILVA; MERCADO, 2018, 2019b). As práticas pedagógicas que utilizam as TICs são vistas como meios oportunos para superar muitos obstáculos no processo de ensino e de aprendizagem. Segundo Sancho (1998)

A prática docente deve responder ás questões reais dos estudantes, que chegam até ela com todas as suas experiências vitais, e deve utilizar-se dos mesmos recursos que contribuíram para transformar suas mentes fora dali. Desconhecer a interferência das tecnologias, dos diferentes instrumentos tecnológicos, na vida cotidiana dos estudantes é retroceder a um ensino baseado na 'ficção' (SANCHO, 1998, p. 40).

A inserção das TICs no ensino de Física tem se mostrado uma prática bastante necessária, mas não recorrente. Para Moreira (2018)

Simulações computacionais, modelagem computacional, laboratórios virtuais deveriam estar naturalmente integrados ao ensino de Física no século XXI. Celulares também poderiam fazer parte dessa tecnologia que deveria permear o ensino de Física nos dias de hoje. Mas não é assim. É claro que a escola pode não ter a instrumentação necessária, mas a principal razão da não incorporação das TIC no ensino de Física na atualidade é 


\section{0 uso do aplicativo ANKI no estudo da termodinâmica no ensino superior: um relato de experiência}

o foco no treinamento para as provas, a ênfase nas "respostas corretas", no emprego de fórmulas para resolver problemas conhecidos. Isso é ensino de Física? Certamente não! (MOREIRA, 2018, p. 76).

$\mathrm{Na}$ literatura encontram-se vários trabalhos teóricos pautados na inserção de laboratórios virtuais e Tecnologias de Informação e Comunicação (AMORIM; DIAS; SOARES, 2015; ARAUJO et al., 2017; CAVALCANTE; TAVOLARO, 2018; DANTAS; PEREZ, 2018; FONSECA et al., 2013; GOMES; CASTRO; ROCHA, 2018; JARDIM, 2017; (LOUREIRO, 2019; OLIVEIRA; PINHEIRO; GOMES, 2019; RIGO, 2014; SILVA; SANTOS; SILVA, 2019; SILVA; MERCADO, 2019a, 2020; VERONEZ et al., 2015).

No Ensino Superior, são muitas as razões que justificam o pouco uso das TICs no ensino de Física, das quais destaca-se a demanda de tempo exigida para aprender a utilizar e para manipular os recursos tecnológicos. É uma reclamação bastante comum, nas falas dos professores de Física do Ensino Superior, o problema gerado pela reduzida carga horária do componente curricular de Física e a extensa lista dos conteúdos programados na ementa do referido componente. Para muitos professores esse fator impede que novas técnicas de ensino sejam aplicadas na prática pedagógica visto que a utilização de novas técnicas demanda maior tempo de aula bem como maior tempo para preparação. Entretanto as contribuições das TICs para o processo de ensino e aprendizagem da Física são incontestáveis.

Atualmente, os smartphones estão cada vez mais cheios de tecnologias e ferramentas de inúmeras utilidades. Existem aplicativos com diversas finalidades, inclusive educativas. Essas propriedades dos smartphones os tornam recursos valiosos na implementação do ensino interativo e contemporâneo aos problemas atuais. Além disso, os smartphones são dispositivos de dimensões reduzidas, portáteis, que podem ser utilizados em qualquer ambiente. 


\section{0 uso do aplicativo ANKI no estudo da termodinâmica no ensino superior: um relato de experiência}

Neste artigo relatamos a nossa experiência e as nossas percepções sobre a utilização do aplicativo ANKI no ensino de Termodinâmica, em uma turma do curso de Agronomia, da Universidade Federal do Cariri (UFCA), localizada no interior do Ceará. Este trabalho é parte do projeto cadastrado em 2019, no Programa de Iniciação a Docência da Pró-reitoria de Graduação da UFCA. O nosso objetivo com a inserção do aplicativo era de avaliar o potencial do aplicativo quanto ao estímulo e a mudança na rotina de estudo dos alunos. Além disso, tínhamos como propósito apresentar aos alunos uma forma uniforme e contínua de estudar, sem picos de stress pré-teste, e de avaliar a percepção dos alunos sobre a inserção do aplicativo na rotina de estudo. A nossa opção de inserir o aplicativo na prática pedagógica se justifica pela praticidade que o recurso oferece aos alunos e ao professor e, pelo potencial que o aplicativo tem de estimular a rotina de estudo, a curiosidade e a criatividade do aluno.

\section{0 aplicativo ANKI}

O aplicativo ANKI é uma ferramenta tecnológica que pode ser utilizada em computadores com sistemas Windows, Mac e Linux e que oferece suporte para imagens, áudio, vídeos e marcação científica (via Latex) (ANKIBRASIL, c2018). O aplicativo também pode ser instalado no smartphone ou acessado via navegador de internet. Talvez uma das propriedades mais expressivas do ANKI seja a possibilidade de usá-lo gratuitamente.

Para compreender o funcionamento básico do aplicativo ANKI é necessário definir alguns conceitos. Entretanto vamos primeiramente falar da chamada Curva do Esquecimento. O conceito da curva do esquecimento foi proposta pelo psicólogo Hermann Ebbinghaus em 1885 (GREEN, 1913). A curva do esquecimento caracteriza como as informações memorizadas são perdidas com o 


\section{0 uso do aplicativo ANKI no estudo da termodinâmica no ensino superior: um relato de experiência}

passar do tempo, caso não haja nenhum tipo de interferência no processo natural de memorização. Salientamos que segundo PERCHER e STEIN (2003) a curva do esquecimento não se comporta da mesma forma para todas as informações.

A título de ilustração, podemos observar a figura 01, na qual temos, no eixo vertical, a porcentagem das informações memorizadas e, no eixo horizontal, o tempo expresso em dias. Na figura 1, a curva de cor preta caracteriza o processo espontâneo de memorização o qual tem um pico no primeiro dia e em seguida decresce exponencialmente com o tempo. Observe que no segundo dia o decréscimo compromete mais da metade da informação recebida inicialmente, correspondendo a uma perda de aproximadamente 75\%, e tende ao completo esquecimento, aproximadamente $90 \%$, nos dias subsequentes. Então surgi a seguinte pergunta: Como contornar essa situação?

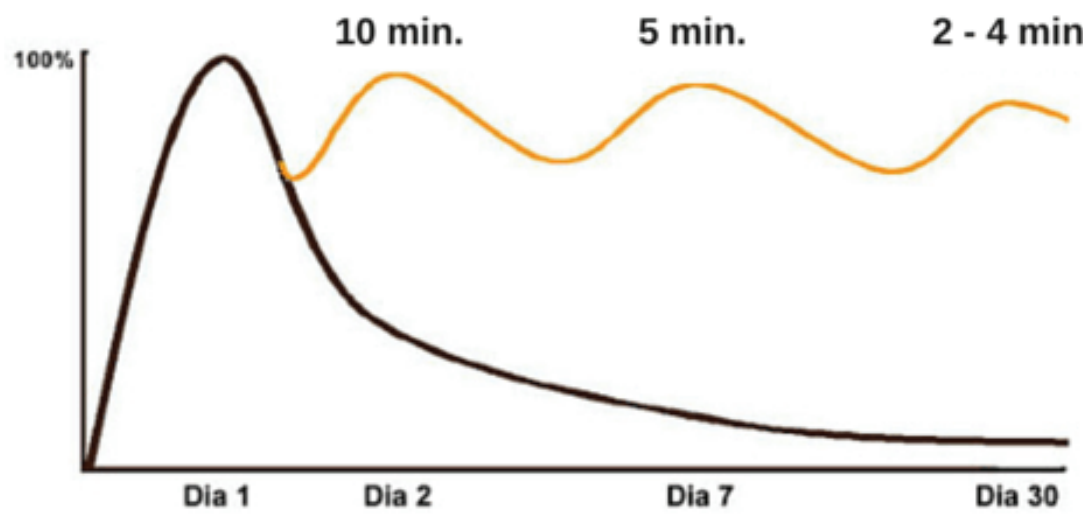

Figura 01: Curva do Esquecimento

Fonte: Figura extraída de (MARQUES, 2016) 


\section{0 uso do aplicativo ANKI no estudo da termodinâmica no ensino superior: um relato de experiência}

Uma possível resposta ou solução do questionamento anterior é o uso de uma técnica chamada de Sistema de Repetição Espaçada (SRE). Essa técnica admiti que existe um momento ideal para conferir ou revisar a informação recebida, principalmente nas primeiras 24 horas, após o recebimento da informação, visto que é nesse período que ocorre de forma mais acentuada o esquecimento da informação. Conforme essa técnica, após o recebimento da informação, serão necessários, no primeiro dia, somente 10 minutos de revisão; no sétimo dia, 5 minutos, e a partir do trigésimo dia apenas de 2 a 4 minutos serão suficientes para manter $100 \%$ da informação armazenada. A caracterização da memorização da informação, com o uso da SRE, está ilustrada na figura 01 com a curva de cor amarela.

Para atender a SRE, a revisão da informação recebida pode ser realizada de forma muito prática e simples utilizando, por exemplo, cartões (chamados de flash-cards) que são pedaços de papel e que geralmente são organizados em blocos formando um baralho. Os cartões são elaborados com temas que o usuário deseja estudar. Com essa finalidade, os cartões são elaborados de modo que, em um lado do cartão, escreve-se uma pergunta ou imagem e no verso, a resposta ou a definição. Existem duas possibilidades quando se examina o cartão: o acerto ou o erro. Havendo erro o cartão deverá ser revisto e por isso deve ser inserido em um baralho (bloco) que exija revisões mais frequentes. Havendo acerto o usuário deverá agrupar o cartão ao baralho que necessita de menos revisão. É ideal que se monte 4 ou 5 baralhos, cada um com uma frequência de revisões sendo o primeiro o que requer revisões mais frequentes e o último, revisões menos frequentes.

Aparentemente, o uso de um baralho para estudar conteúdos é facilmente aplicável quando o número de cartões é relativamente pequeno. Entretanto quando há necessidade de muitos cartões, centenas ou até milhares, a praticidade da técnica perde o sentido. Nesse caso o uso dos cartões perde uma das suas maiores vantagens que é a possibilidade de consulta-los em qualquer lugar e 


\section{0 uso do aplicativo ANKI no estudo da termodinâmica no ensino superior: um relato de experiência}

sempre que tiver oportunidade. Além disso, como os cartões são feitos à mão, o que nele é inserido fica limitado ao que o usuário pode escrever ou desenhar. A técnica que utiliza os cartões para o aprendizado acelerado e potencializado pelo SRE consiste no Sistema Leitner que foi desenvolvido por Sebastian Leitner na década de 70.

Uma ótima ferramenta para sanar as dificuldades dos cartões de papel, é o uso de uma ferramenta digital, por exemplo, o aplicativo ANKI. Utilizando o aplicativo ANKI é possível elaborar cartões digitais e montar baralhos específicos de cada conteúdo. Os cartões elaborados pelo ANKI são dotados de perguntas e respostas e quando a resposta é visualizada em tela, a pergunta permanece em tela e o usuário informa ao programa como conseguiu responder a pergunta, clicando nas seguintes opções - Novamente (se o usuário errou), Difícil, Bom, Fácil. O programa usa esse feedback para decidir o momento adequado para mostrar os cartões do baralho. O cartão para o qual o usuário atribuiu o termo "Novamente" será mostrado em seguida e, posteriormente, será mostrado com intervalos menores que os intervalos para os cartões classificados como "Fácil" ou "Bom". Aqueles cartões para os quais o usuário atribuiu o termo "Difícil" também serão mostrados com intervalos menores. Os cartões são elaborados por um usuário e podem ser disponibilizados para outros usuários, mas a montagem do baralho é realizada pelo aplicativo e, para isso, o aplicativo mapeia o desempenho do usuário. O aplicativo conta também com uma aba denominada Estatística na qual o usuário pode verificar o seu desempenho incluindo número de cartões estudados em um determinado tempo, tempo gasto no estudo dos cartões, previsão de revisões dos cartões e intervalos entre as revisões.

Aparentemente, o uso dos cartões elaborados por meio do aplicativo ANKI constitui uma boa opção para sanar os problemas recorrentes da curva do esquecimento visto que o aplicativo pode ser acessado pelo smartphone ou pelo computador, sem necessidade de conexão a internet, a qualquer momento. Assim, os cartões podem ser acessados em conformidade com a SRE. Em 


\section{0 uso do aplicativo ANKI no estudo da termodinâmica no ensino superior: um relato de experiência}

particular, o ANKI parece ser uma excelente alternativa para os alunos de graduação os quais, em geral, cursam um número significativo de componentes curriculares por semestre letivo e talvez gerenciar o tempo ideal (observando a SER) para fazer a revisão dos vários conteúdos não seja uma tarefa fácil. Além disso, os alunos de graduação passam a maior parte do dia na Universidade, não tendo condições de transportar um número expressivo de materiais para os seus estudos, inclusive, os cartões de papel.

\section{Metodologia}

O Projeto de Iniciação a Docência foi desenvolvido no campus Crato da Universidade Federal do Cariri (UFCA), no componente curricular Física Básica II, do curso de Agronomia. O campus Crato da UFCA localiza-se no município de Crato o qual possui aproximadamente 135.604 habitantes segundo o IBGE de 2017 e situa-se no interior do Ceará, a uma distância de $508 \mathrm{~km}$ da capital cearense. A turma era composta por 10 alunos os quais já cursaram entre $50 \%$ e $75 \%$ do curso de Agronomia. Do quantitativo total de alunos, 33,3\% estava repetindo o componente curricular e 66,7 estava tendo o primeiro contato com o referido componente.

O componente curricular Física Básica II, na grade curricular do curso de Agronomia da UFCA, abrange conteúdos de Fluidos, Termodinâmica e Oscilações. Para a utilização do aplicativo escolhemos o conteúdo de Termodinâmica, mas precisamente, Calorimetria e a Primeira Lei da Termodinâmica. A escolha se justifica pela relevância do conteúdo para o estudo de conteúdos subsequentes e mais complexos que exigem uma base conceitual sólida, por exemplo, a segunda da Termodinâmica, e pelo viés conceitual do conteúdo. Após a escolha do conteúdo, discutimos sobre as possíveis técnicas que poderiam ser capazes de estimular os alunos a estudarem com mais 


\section{0 uso do aplicativo ANKI no estudo da termodinâmica no ensino superior: um relato de experiência}

ânimo e frequência. Em virtude da aproximação entre os estudantes e os smartphones optamos pelo uso de um aplicativo. Dentre os vários aplicativos existentes, optamos pelo ANKI, em virtude da técnica do SRE, da possibilidade de elaboração dos cartões digitais para compor o baralho, da facilidade e da praticidade de utilização do aplicativo.

Os cartões para compor o baralho com o conteúdo escolhido foram elaborados no aplicativo ANKI em sua versão 2.1.15 para computador Windows. Embora os cartões tenham sido elaborados através da versão para computador, os alunos acessaram os cartões via versão para smartphone. Com a finalidade de criar cartões com qualidade conceitual e didática consultamos diversos livros na área do conteúdo (HALLIDAY; RESNICK; Walker, 2013; HEWITT, 2011; HUSSENZVEIG, 2014; YOUNG ; FREEDMAN, 2008). O processo de criação do cartão é relativamente simples e contem basicamente os seguintes passos:

- Ao acessar o aplicativo, visualiza-se na parte superior da tela 05 (cinco) abas: Baralhos, Adicionar, Painel, Estatísticas, Sincronizar.

- Na parte inferior da tela visualiza-se 03 (três) abas: Obter Compartilhado, Criar baralho, Importar arquivo. Clicando na aba Criar Baralho, abrirá uma janela na qual deverá ser inserido o nome do baralho.

- Após a criação do baralho, o próximo passo é adicionar os cartões. Para criar e adicionar os cartões clicase na aba “Adicionar" e, em seguida abrirá uma janela contendo duas caixas, que representam a frente e verso do cartão. A pergunta deve ser inserida no campo Frente e a resposta no campo Verso.

Elaboramos um total de 02 (dois) baralhos e 17 (dezessete) cartões envolvendo calorimetria e a Primeira Lei da Termodinâmica. Procuramos elencar perguntas de cunho conceitual e matemático 


\section{0 uso do aplicativo ANKI no estudo da termodinâmica no ensino superior: um relato de experiência}

para que os alunos pudessem refletir sobre as aplicações práticas dos conteúdos estudados e pudessem identificar o aspecto interdisciplinar da Termodinâmica. O nosso baralho de Calorimetria e da Primeira Lei da Termodinâmica foi elaborado com 07 (sete) cartões cujas perguntas estão listadas no quadro 1.

Quadro 1 - Perguntas para elaboração do Baralho de Calorimetria e da Primeira Lei da Termodinâmica.

\begin{tabular}{|c|c|c|}
\hline Ordem & Calorimetria & Primeira Lei \\
\hline 01 & $\begin{array}{l}\text { Qual é a relação entre as escalas de temperatura } \\
\text { Celsius e Kelvin? }\end{array}$ & Defina um processo reversível. \\
\hline 02 & $\begin{array}{l}\text { Qual é a relação entre as escalas de temperatura } \\
\text { Celsius e Fahrenheit? }\end{array}$ & O que afirma a Primeira Lei da Termodinâmica? \\
\hline 03 & $\begin{array}{l}\text { Qual é a relação entre as unidades de medidas } \\
\text { Pascoal e Atmosferas? }\end{array}$ & $\begin{array}{l}\text { Qual é a relação conceitual e matemática entre o calor } \\
\text { específico a volume constante e à pressão constante. }\end{array}$ \\
\hline 04 & $\begin{array}{l}\text { Como se expressa matematicamente a } \\
\text { expansão linear, superficial e volumétrica de } \\
\text { um corpo? }\end{array}$ & $\begin{array}{l}\text { Escreva a expressão do calor específico de um gá } \\
\text { monoatômico, diatômico e poliatômico. }\end{array}$ \\
\hline 05 & O que afirma a Lei Zero da Termodinâmica? & $\begin{array}{l}\text { Escreva a forma da Primeira Lei para os processos } \\
\text { cíclicos, adiabático, isocórico e isobárico. }\end{array}$ \\
\hline 06 & $\begin{array}{l}\text { Quais são as variáveis termodinâmicas do } \\
\text { processo de transferência de energia? }\end{array}$ & $\begin{array}{l}\text { Cite } 3 \text { (três) exemplos de aplicação da Primeira Lei } \\
\text { da Termodinâmica na Agronomia. }\end{array}$ \\
\hline 07 & Defina calor. & $\begin{array}{l}\text { Cite } 3 \text { (três) exemplos de aplicação da Primeira Lei } \\
\text { da Termodinâmica em suas atividades diárias. }\end{array}$ \\
\hline 08 & Defina Calor específico & - \\
\hline 09 & $\begin{array}{l}\text { Cite exemplos de aplicação da Calorimetria na } \\
\text { Agronomia. }\end{array}$ & - \\
\hline 10 & $\begin{array}{l}\text { Cite exemplos da Calorimetria nas suas } \\
\text { atividades diárias. }\end{array}$ & - \\
\hline
\end{tabular}

Fonte: Elaborado pelos autores (2021). 


\section{0 uso do aplicativo ANKI no estudo da termodinâmica no ensino superior: um relato de experiência}

Após a elaboração dos cartões, apresentamos o aplicativo aos alunos e explicamos como gostaríamos de inseri-lo no estudo do componente curricular. Imediatamente os alunos demostraram interesse e curiosidade sobre o aplicativo. Em seguida estabelecemos em comum acordo uma data para a implementação da proposta e um período para sua execução, que foi de 4 (quatro) semanas. No período de execução da proposta, os cartões eram disponibilizados em etapas conforme o andamento da aula e a evolução da abordagem do conteúdo. Reforçamos que não era a nossa proposta estabelecer a frequência de acesso ao aplicativo por parte dos alunos, mas sim de avaliar o estímulo do aplicativo sobre a rotina de estudo do aluno e consequentemente, suas contribuições para o processo de aprendizagem naquele grupo de alunos particular. Por essa razão, deixamos em aberto como e quando cada aluno interagiria com o aplicativo.

\section{Resultados}

Quando concluímos as 04 (quatro) semanas de aplicação da proposta, disponibilizamos um Questionário de Avaliação da Proposta, não identificado, elaborado pelos autores. Dos 10 alunos matriculados no componente curricular e que tiveram acesso ao nosso baralho, 05 compuseram a nossa amostra. Por simplicidade enumeramos os questionários por ordem de recebimento e nesse processo de análise dos resultados nos referiremos aos alunos como aluno 01, aluno 02 e assim sucessivamente. O questionário continha 07 (sete) perguntas, em sua maioria, de múltipla escolha. Perguntamos, primeiramente, o período letivo no qual os alunos estavam matriculados e percebemos que todos os alunos já haviam cursado de 50\% a 75\% do curso de Agronomia. É importante ressaltar que o componente curricular Física Básica II é oferecido no segundo semestre do curso, mas por várias razões os alunos acabam cursando somente no final do curso. 


\section{0 uso do aplicativo ANKI no estudo da termodinâmica no ensino superior: um relato de experiência}

A segunda pergunta do questionário visava verificar a porcentagem de alunos que estavam repetindo o componente curricular e, para aqueles que estavam repetindo, quais as possíveis razões da não aprovação ou da desistência do componente curricular quando cursado anteriormente. Os resultados do questionário apontaram que 33,3\% dos alunos cursaram o componente curricular em um momento anterior, porém sem obter êxito. Essa parcela dos alunos atribuiu a dificuldade de assimilar o conteúdo como justificativa da não aprovação ou da desistência do componente curricular cursado no momento anterior.

Os alunos foram questionados quanto à influência do aplicativo sobre o aprendizado do conteúdo estudado e todos consideraram que o aplicativo influenciou positivamente. Ao responder o questionário, os alunos tinham 05 opções para classificar a influência do aplicativo, quais sejam Ótima, Boa, Regular, Ruim e Péssima. Do total de participantes, 66,7\% avalia a influência do aplicativo sobre o aprendizado como ótima enquanto que 33,3\% considera que a influência do aplicativo é boa.

Por meio do questionário verificamos o potencial do aplicativo sobre a motivação para estudar o conteúdo proposto. Foram fornecidas 05 respostas que indicavam o grau de concordância do aluno quanto à motivação para estudar o conteúdo e 50\% concordaram que se sentiram parcialmente motivados a estudar com o aplicativo e os outros 50\% apontaram que se sentiram totalmente motivados a estudar após a introdução do aplicativo em sua rotina de estudo.

Solicitamos também que os alunos elencassem as contribuições e os desafios encontrados na implementação do aplicativo em sua rotina de estudo. Conforme as respostas, os alunos consideraram que as contribuições eram relativas à evolução do processo de compreensão, assimilação e memorização dos conteúdos. A praticidade e a facilidade de acesso também foram apontadas como pontos positivos do aplicativo que contribuem para sua inserção na rotina de 


\section{0 uso do aplicativo ANKI no estudo da termodinâmica no ensino superior: um relato de experiência}

estudo. Percebemos que as respostas fornecidas corroboram com a avaliação, por parte dos alunos, sobre a influência do aplicativo sobre o aprendizado e com a avaliação da motivação do aplicativo para estudar o conteúdo. Na concepção dos alunos a dificuldade de download do baralho para alguns dispositivos foi caracterizada como um desafio da proposta. Salientamos que esse foi o único desafio apontado.

A avaliação quantitativa do potencial do aplicativo quanto a fixação do conteúdo estudado mostrou que $100 \%$ dos alunos atribuíram notas maiores ou iguais a 8 . Mais especificamente, 33,3\% atribuíram nota $10,16,7 \%$ atribuíram nota 9 e 50\% atribuíram nota 8 .

\section{Considerações finais}

$\mathrm{Na}$ literatura existe um número significativo de trabalhos que abordam o uso das TICs como meios de agregar interatividade, diversão e dinamismo às aulas dos variados componentes curricular da Educação Básica e Superior. Em particular no ensino de Física aborda-se veemente o uso dos aplicativos como alternativa valiosa na superação das dificuldades encontradas no processo de ensino e de aprendizagem. Neste artigo procuramos avaliar e incentivar a implementação do aplicativo ANKI na rotina individual de estudo dos alunos do Ensino Superior, particularmente, no estudo da Termodinâmica. Certamente a intervenção na rotina de estudo dos alunos refletirá no processo de ensino e de aprendizagem.

Analisando as respostas do Questionário de Avaliação da Proposta e o desenvolvimento dos alunos verificamos que o aplicativo ANKI constitui um recurso potencialmente estimulante para o Ensino Superior. Acreditamos que a proposta deva ser aplicada a outros conteúdos, outros componentes curriculares e até mesmo a outros cursos do Ensino Superior. Talvez o período 


\section{0 uso do aplicativo ANKI no estudo da termodinâmica no ensino superior: um relato de experiência}

epidemiológico, de ensino remoto, no qual nos encontramos seja bastante adequado para a implementação do aplicativo ANKI na rotina de estudo tendo em vista as suas potencialidades quanto a interação virtual. Outro ponto que nos leva a incentivar o uso do aplicativo ANKI é a liberdade e a facilidade que o professor tem de criar seu próprio baralho observando outras estratégias de ensino, o perfil dos seus alunos e o perfil do curso.

\section{Referências}

AMORIM, H. S.;DIAS, M. A. ; SOARES, V. Sensores digitais de temperatura com tecnologia one-wire: um exemplo de aplicação didática na área de condução térmica. Revista Brasileira de Ensino de Física, v. 37, n. 4, p. 1 - 9, 2015.

ANKI BRASIL. Inteligentes e poderosos Flashcards. Lembrar ficou ainda mais fácil. c2018. Página inicial. Disponível em: https://www.ANKIbrasil.com/. Acesso em: 30 de nov. de 2020. 2018.

ARAÚJO, F. A. G. et. al. O estudo de dinâmica dos fluidos com o aplicativo Wind Tunnel: analisando o voo de um avião. Revista do Professor de Física, v. 1, n. 2, p. 25-36, 2017.

CAVALCANTE, M. A.; TAVOLARO, C. R. C. Espectroscopia com novas tecnologias: o tracker como ferramenta. Revista do Professor de Física, v. 2, n. 3, p. 121-130, 2018.

DANTAS, M. ; PEREZ, S. Gamificação e jogos no ensino de mecânica newtoniana: uma proposta didática utilizando os aplicativos Bunny Shooter e Socrative. Revista do Professor de Física, v. 2, n. 2, p. $84-103,2018$.

FONSECA, M. et. al. O laboratório virtual: uma atividade baseada em experimentos para o ensino de mecânica. Revista Brasileira de Ensino Física, v. 35, n. 4, p. 1-10, 2013. 


\section{0 uso do aplicativo ANKI no estudo da termodinâmica no ensino superior: um relato de experiência}

GOMES, E. C.; CASTRO, W. S. ; ROCHA, A. S. O ensino de Física interativo: Blog, ferramenta de aprendizagem no século XXI. Experiência em Ensino de Ciências, v. 13, n. 1, p. $154-168,2018$.

GREEN, C. D. Memory: A contribution to experimental psychology. Classics in the history of psychology. Disponível em: <http://psychclassics.yorku.ca/Ebbinghaus/index. htm>. Acesso: 30 de nov. de 2020. 1913.

HALLIDAY, D.; RESNICK, R. ; Walker, J. Fundamentos de Física: Mecânica. v. 1. 9. ed. Rio de Janeiro: LTC. 2013.

HEWITT, P. G. Física Conceitual. 11. ed. Porto Alegre: Bookman. 2011.

HUSSENZVEIG, H. M. Curso de Física Básica. v. 2. 5. ed. São Paulo: Blucher. 2014.

JARDIM, D. F. O. laboratório virtual como espaço para aprendizagem de conteúdo de análise dimensional - um relato de experiência do uso do GeoGebra no ensino de Física. Vozes dos Vales. V. 5, n. 11, p. 1 - 19, 2017.

LOUREIRO, B. C. O. O uso das tecnologias da informação e comunicação como recursos didáticos no ensino de Física. Revista do Professor de Física, v. 3, n. 2, P. 93 $102,2019$.

MARQUES, W. Técnica de revisão: entenda a curva do esquecimento. VestGeek, 2020. Disponível em: <https://www.vestgeek.com/tecnica-revisao-curva-esquecimento-enem/>. Acesso: 30 de nov. de 2020, 2016.

MOREIRA, A. Uma análise crítica do ensino de Física. Estudos Avançados, v. 32, n. 94, p. $73-80,2018$.

NUNES, E.; SILVA, I. P. ; MERCADO, L. P. L. Levantamento dos temas TIC e EAD nos periódicos qualis. Informática na Educação: teoria e prática, v. 20, n. 2, p. 15 - 34, 2016. 


\section{0 uso do aplicativo ANKI no estudo da termodinâmica no ensino superior: um relato de experiência}

OLIVEIRA, E. R.; PINHEIRO, A. R. C. ; GOMES, I. F. Aplicativo com fenômenos do cotidiano para desenvolvimento dos conceitos de Física Moderna, Revista do Professor de Física, Brasília, v. 3, (especial). P. $79-80,2019$.

PERGHER, G. K. ; STEIN, L. M. Compreendendo o esquecimento: teorias clássicas e seus fundamentos experimentais. Psicologia USP, v. 14, n. 1, p. 129 - 155, 2003.

RIGO, J. R. V. Um olhar sobre o uso das TIC no ensino de Física. 2014. 84f. Dissertação (Mestrado Profissionalizante em Ensino de Física e Matemática) - Centro Universitário Franciscano, Santa Maria.

SANCHO, J. M. Para uma Tecnologia Educacional. (Tradução Beatriz Afonso Neves). Porto Alegre, Artmed, 1998.

SEABRA, M. E. F. ; MACIEL, A. M. M. Ensino de Física por projeto: o estudo de termologia em sala de aula favorecendo a alfabetização científica. Experiência em Ensino de Ciências, v. 14, n. 1, p. $330-343,2019$.

SILVA, I. P. ; MERCADO, L. P. L. Levantamento de dados acerca do tema “Experimentação Mediada por Interfaces Digitais (2005 - 2015). Revista Paidei@, v. 10, n. 17, p. 1 - 25, 2018.

SILVA, I. P.; MERCADO, L. P. L. Experimentação em Física apoiada por objetos de aprendizagem. ACTIO, v. 4, n. 2, p. 71-86, 2019a

SILVA, I. P. ; MERCADO, L. P. L. Revisão sistemática de literatura acerca da experimentação virtual no ensino de Física. Ensino e Pesquisa, v. 17, n. 1, p. 49-77, $2019 \mathrm{~b}$

SILVA, I. P. ; MERCADO, L. P. L. Laboratórios de ensino de Física mediados por interfaces digitais. EDUCA - Revista Multidisciplinar em Educação, v. 7, n. 17, p. 3 - 22, 2020. 


\section{0 uso do aplicativo ANKI no estudo da termodinâmica no ensino superior: um relato de experiência}

SILVA, K. W.; SANTOS, B. M. ; SILVA, L. R. Utilização de apps para o ensino do efeito Doppler. Revista do Professor de Física, v. 3(Especial)p. 89-90, 2019.

VERONEZ, D.; et. al. A utilização das TICs no ensino de Física para trabalhar conceitos de MRU e MRUV. Ensino e Pesquisa, v. 13, n. 1, p. 152 - 165, 2015.

YOUNG, H. D. \& FREEDMAN, R. A. Física I: Mecânica. 12. ed. São Paulo: Pearson. 2008. 